Fecha de recepción: marzo 2020 Fecha de aceptación: abril 2020 Versión final: junio 2020

\section{De la disputa a la colaboración mediático-política en Ecuador. Análisis comparado de los frames mediáticos en las protestas de 2015 y 2019}

Palmira Chavero ${ }^{(1)}$

\begin{abstract}
Resumen: La última década en Ecuador estuvo marcada por la disputa mediático-política entre el entonces presidente de la República, Rafael Correa, y los medios de comunicación privados que llegaron a convertirse en actores políticos. Con la llegada en 2017 del nuevo presidente, Lenín Moreno (fungido sucesor por el propio Correa), Ecuador enfrentó un cambio no sólo de líder, sino también un giro brusco en la política nacional. Las fuerzas políticas y económicas conservadoras, adversarias durante la presidencia de Correa, se convirtieron con Moreno en aliadas. En ese giro, los medios de comunicación pasaron, asimismo, de ser adversarios y disputar el terreno político a Correa a convertirse en aliados de Moreno.

Este artículo realiza un análisis comparado de la manera en que dos de los principales medios encuadraron las reacciones sociales a distintas medidas económicas: las leyes de Herencia y Plusvalía de Correa (2015) y el Decreto 883 de Moreno (2019). Se toman para ello dos medios, El Telégrafo (público) y El Comercio (privado), ya que permiten identificar las diferencias entre los medios en función de su naturaleza jurídica en cada período.
\end{abstract}

Palabras clave: frames - mediatización de la política - medios de comunicación masiva - protestas - Ecuador.

[Resúmenes en inglés y portugués en la página 49]

(1) Es Doctora en Ciencias de la Comunicación y Sociología por la Universidad Complutense de Madrid (España). Investigadora del equipo de investigación Agenda y Voto y del Grupo de Investigación en Gobierno, Administración y Políticas Públicas (GIGAPP). Profesora titular e investigadora en la Facultad Latinoamericana de Ciencias Sociales (FLACSO) Sede Ecuador. Coordinadora de la Maestría de investigación en Comunicación y Opinión Pública de FLACSO Ecuador. Directora de FLACSO Radio. Ha impartido clases en la Universidad Complutense de Madrid (España), el Instituto Universitario de Investigación Ortega y Gasset (España) y ha sido coordinadora del Laboratorio de Comunicación y Derechos en Ecuador de Ecuador (IAEN). 


\section{Introducción}

Las últimas décadas en América Latina han estado marcadas por gobiernos de signo progresista, caracterizados en algunas ocasiones como populistas, entre cuyas acciones ha destacado un fuerte activismo estatal (Fiss, 1997) en materia de comunicación mediática, lo que ha originado en muchos casos la elaboración de políticas públicas en esa materia. Esta intervención del Estado ha modificado los sistemas mediáticos (Hallin y Mancini, 2008) característicos de la región y ha introducido cambios en las relaciones entre los actores políticos (gobiernos, fundamentalmente) y los mediáticos.

En los últimos años, sin embargo, asistimos a un nuevo cambio en esta materia, con un retorno a la autorregulación y una disminución de la conflictividad que hasta ahora predominaba en la relación entre estos actores. Esta nueva (o vieja) relación entre los poderes político y mediático resulta particularmente significativa en un momento en el que se están tomando medidas impopulares que tendrían un alto costo político sin el apoyo de los grandes medios de comunicación de un país. Por tanto, analizar este posible cambio en las relaciones mediático-políticas se torna un elemento de interés no sólo para los estudios de Comunicación Política, sino incluso para entender la procesos sociales y políticos que se están desarrollando en la actualidad en América Latina.

\section{Los frames en los procesos de mediatización de la política}

Tradicionalmente en los estudios de comunicación, a la hora de analizar el sistema mediático de un país, se ha acudido a la propuesta realizada por Hallin y Mancini (2008), en la que establecen tres tipos ideales de sistemas mediáticos en función de la interacción que se produce entre el mercado, el Estado y los medios de comunicación. Según la propuesta de estos autores, la configuración de un sistema mediático es el resultado de determinadas características: industria de la prensa, paralelismo político, profesionalización y autonomía de los periodistas y papel del Estado. La combinación de estos factores da origen, según los autores, a tres modelos típicos de sistemas mediáticos; el modelo liberal, el democrático corporativo y el pluralista polarizado.

Estos modelos no sólo tienen la limitación de tratarse de tipos ideales a los que habría que incorporar matices, sino que sus propios autores reconocen que los modelos podrían estar cambiando. Además, la propuesta de Hallin y Mancini adolece de estar basada en países europeos y norteamericanos que, si bien pueden compartir algunas características con América Latina, gozan de una democracia relativamente consolidada y quedan lejos de la idiosincrasia latinoamericana (Hallin y Papathanassopoulos, 2002). Autores como Albuquerque (2012) reclaman la ruptura de la dependencia de la visión occidental para el análisis de países con democracias emergentes.

En el caso de América Latina, el sistema mediático se caracteriza por unos fuertes niveles de concentración empresarial histórico, que sigue pesando: impulso a los medios comunitarios, intervención del Estado, baja profesionalización, escasa penetración aún de las TIC (aunque es una tendencia en aumento) pero un mercado eminentemente audiovi- 
sual y una fuerte crisis de confianza en los medios (Chavero y Oller, 2015). Algunos autores definen el sistema mediático como "liberal capturado" (Guerrero y Márquez, 2014), en el que tendríamos un desarrollo inicial de propiedad privada en estrecha relación con el poder político y, ya en el siglo XXI, una confrontación entre los grandes grupos privados y los gobiernos progresistas, quienes han tomado la iniciativa en la regulación del sector mediático.

En la actualidad, sin embargo, parece haber vuelto la tendencia inicial: recuperación del protagonismo de los grupos privados y consonancia con el poder político, lo que se ve reflejado en una mayor gubernamentalización de los medios públicos, eliminación de la redistribución equitativa del espectro radioeléctrico y recuperación de los privilegios de los grandes grupos (Chavero, 2018).

Las características de los diferentes tipos de sistemas mediáticos, en especial lo referido al paralelismo político y -en menor medida- la profesionalización, se ven reflejadas no sólo en el posicionamiento de los medios y su relación con el poder sino, más en concreto, en el contenido mismo. De esta manera, podemos observar procesos de mediatización en los que los medios de comunicación abandonan su rol de mediadores y tratan de interferir en la agenda política, tomando con ello un papel de actores políticos. Estos procesos de mediatización (Mazzoleni y Schulz, 1999) se suelen desarrollar en cuatro fases con distintos grados (Strömback, 2008), en las que además se pueden identificar distintos indicadores: el uso de los medios como fuente principal de información política, la dependencia del poder político o la asunción de las lógicas mediáticas por los actores políticos (Strömback, 2008).

En la práctica, un proceso de mediatización pasa por tres momentos: la definición de la agenda temática, el enmarcamiento o framing de los principales temas y el debate público sobre los mismos, en el que no es infrecuente que intervengan actores adicionales (Chavero, 2015). En el primer momento, los medios de comunicación seleccionan una serie de temas, que proponen como los más relevantes y que pasan a ser parte de la agenda pública (McCombs, 2004). Estos temas son convenientemente enmarcados (Entman, 1993) y, en último término, se convierten en objeto de debate público. En este proceso, es particularmente relevante el papel que desempeñan los medios a la hora de encuadrar cada uno de los temas, entendido el framing como el proceso de "seleccionar algunos aspectos de una realidad que se percibe y darles más relevancia en un texto comunicativo, de manera que se promueva una definición del problema determinado, una interpretación causal, una evaluación moral y/o una recomendación de tratamiento para el asunto descrito" (Entman, 2004, p. 5). Esta propuesta inicial se corresponde con las funciones que el mismo autor asigna a los encuadres o frames y que permiten, asimismo, ver su eficacia en el debate público: 


\begin{tabular}{|c|c|c|c|}
\hline \multicolumn{4}{|c|}{ Foco del encuadre } \\
\hline Función del encuadre & Issues & Events & $\begin{array}{c}\text { Actores politicos } \\
\text { (individual, grupos, } \\
\text { naciones) }\end{array}$ \\
\hline \multicolumn{4}{|c|}{$\begin{array}{l}\text { Definición de los efectos/condiciones } \\
\text { problemáticos }\end{array}$} \\
\hline \multicolumn{4}{|l|}{ Identificación de la causa/agente } \\
\hline \multicolumn{4}{|l|}{ Propuesta de solución } \\
\hline Comunicar juicios morales & & & \\
\hline
\end{tabular}

Figura 1. Funciones y objetos de los encuadres. Fuente: Entman (2004, p. 24).

No es infrecuente que el encuadre (frame) que el medio ofrece del tema vaya acompañado, además, de una serie de atributos con los que caracteriza a los actores asociados.

\section{El caso de Ecuador, preguntas e hipótesis}

El escenario mediático latinoamericano en las últimas décadas ha estado caracterizado por la mayor intervención del Estado en países con gobiernos denominados populistas. En el caso de Ecuador, la Ley de Orgánica de Comunicación (LOC) fue una promesa de Rafael Correa en su primera campaña electoral, que vio la luz en junio de 2013 en la Asamblea Nacional. El Estado ecuatoriano viene actuando en materia de comunicación en otros aspectos: como actor económico, como regulador de contenidos mediáticos, como regulador de la propiedad o como agente de formación y capacitación, entre otros (Chavero 2015b). Con el cambio de gobierno en 2017 y el giro protagonizado por el nuevo presidente, una de las primeras propuestas de reformas fue, precisamente, la de la Ley Orgánica de Comunicación, reforma fuertemente demandada por los principales medios privados. En 2019, después de un proceso en el que el Ejecutivo observó su propia propuesta, se aprueba la reforma a la LOC, en la que destacan algunos elementos que rompían con el espíritu de la norma anterior. En la nueva ley se elimina la Superintendencia de Comunicación (Supercom), así como todos los elementos sancionatorios de la norma y la obligatoriedad de que los medios cuenten con un código y normas de carácter deontológico (Art. 9 y 10 anterior LOC). En lo que se refiere a la distribución por tercios del espectro radioeléctrico, la nueva ley reduce el espacio reservado para los medios públicos en favor de los medios privados, de manera que éstos recuperan, por ley, el predominio de la propiedad:

Se reservará hasta el 34\% del espectro radioeléctrico al sector comunitario en función de la demanda y de la disponibilidad, porcentaje máximo que deberá alcanzarse progresivamente. El 66\% del espectro restante será asignado para el sector público y privado en función de la demanda, no debiendo exceder la 
asignación de frecuencias al sector público un porcentaje del 10\% del espectro (Art.87, Reforma LOC).

En esta línea, modifica la definición de medios públicos y elimina los objetivos, además se abre la vía a la comercialización de sus productos como forma de financiación. También se modifica la definición de medios privados, eliminando la "responsabilidad social", así como la de los medios comunitarios.

En un acercamiento a los grandes medios privados, la nueva LOC incorpora una serie de articulados referidos a la autorregulación de los medios de comunicación (Art. 91.1 a 91.4) y elimina la prohibición de censura previa de "accionistas, socios o anunciantes" (Art. 18 anterior LOC) limitándose la prohibición de censura a los funcionarios públicos (Art. 11); también se reduce la responsabilidad de los medios (Art. 20 y 21) y desaparece la obligatoriedad de la disculpa pública en caso de tener que rectificar (Art. 23), así como la obligatoriedad de difundir el tiraje. La obligatoriedad de proteger a los trabajadores se reduce a aquellos "que estén en riesgo".

En lo que respecta a la relación con los ciudadanos, se elimina la conceptualización de "democratización de la propiedad y acceso a los medios de comunicación" (Art. 12), que era una de las consignas de la ley anterior y, en su lugar, se apuesta por una "comunicación democrática". También se sustituye el derecho a recibir información "de relevancia pública veraz" por "información de calidad" y se deroga el artículo que garantiza la "libertad de información” (Art. 29). Por último, se elimina la figura del defensor de las audiencias y todas las funciones de protección al ciudadano que recaían sobre la Supercom pasan a ser jurisdicción de la Defensoría del Pueblo.

Además de estas reformas a la norma, en diciembre de 2019 se elimina el pago del IVA al papel de periódico en la Ley Orgánica de Simplicidad y Progresividad Tributaria (Art.55.7), que reformaba la Ley Orgánica de Régimen Tributario Interno (LORTI)1 .

Con estas medidas, lo que antes era disputa del poder político con los grandes medios de comunicación ahora se torna nuevamente acercamiento a ellos; esta nueva relación podría significar, en consecuencia, un retorno a la colaboración mediático-política en Ecuador, lo cual sería particularmente significativo en momentos de convulsión social o reformas estructurales. A partir de ello, nos preguntamos cómo ha cambiado la relación de los medios de comunicación en Ecuador con los dos gobiernos: el de Rafael Correa y el de Lenín Moreno.

\section{Hipótesis y metodología}

Para responder a la pregunta anterior, centramos nuestro análisis en dos momentos de especial relevancia, en los que cada uno de los Presidentes toma medidas que generan protestas sociales. El primero de los momentos responde a una serie de marchas que la oposición y algunos grupos protagonizaron en junio de 2015 para protestar por las Leyes de Herencias y Plusvalía que propuso Rafael Correa. El segundo momento es el paro nacional protagonizado por los movimientos sociales en octubre de 2019 como respuesta 
al Decreto 883, que contenía una serie de medidas de ajuste entre las que destacaba el aumento del precio del combustible.

La comparación de la cobertura en los dos momentos nos permitirá identificar si, en efecto, hubo un cambio en la relación entre los medios de comunicación y los distintos gobiernos. Atendiendo a las particularidades del sistema mediático ecuatoriano, en especial en lo relativo al paralelismo político, consideramos interesante realizar este estudio a través de la comparación de dos de los principales medios de comunicación impresa de Ecuador, uno público (El Telégrafo) y otro privado (El Comercio). Esto nos permitirá identificar posibles diferencias entre ellos en ambos momentos.

Para desarrollar este análisis, realizamos un análisis de framing de las portadas y los editoriales de los dos períodos mencionados, a partir de la propuesta de Entman. En concreto, analizamos desde el 25 de mayo al 25 de junio de 2015 y del 1 al 20 de octubre de 2019. La hipótesis (H1) de la que partimos es que, mientras en 2015 los medios privados hicieron de oposición al gobierno y protagonizaron un proceso de mediatización, en 2019 los medios de comunicación privados se alinearon con el gobierno en una muestra de colaboración. En segundo lugar (H2), proponemos que, fruto de esta colaboración político-mediática, en 2019 se da una mayor gubernamentalización de los medios públicos de Ecuador.

\section{Principales resultados}

\section{Las marchas de las banderas negras (2015)}

El 24 de mayo de 2015, en el Informe anual a la Nación, el entonces presidente Rafael Correa anunció una nueva ley que gravaría las herencias: "Enfrentaré el costo político que sea necesario, pero en los próximos días también enviaré a la Asamblea Nacional un nuevo sistema impositivo para las herencias superiores a los cien salarios básicos unificados". En la práctica, esto supondría un nuevo impuesto para herencias superiores a los 50.000 dólares, que afectaría, según el Gobierno, al 2\% más rico de la población ecuatoriana.

Frente a este anuncio, en el que no se detallaba mucho más, la prensa privada reacciona:

Está claro que un patrimonio tan magro no es, ni mucho menos, privilegio de las clases que ocupan los quintiles superiores de la población. Es quizá un patrimonio que se hereda de familias de clase media, al menos. Entonces la redistribución no funciona. Debe revisarse la cifra con argumentos técnicos (Editorial El Comercio, 27/05/2015).

Esta posición recibió el apoyo de otros medios privados: "Este impuesto, que no es de ninguna manera para los ricos, afectará a un gran porcentaje de la población" (Editorial El Universo, 27/05/2015) y también de otros actores, como los empresarios, que expresaron su rechazo a esta medida y a la relativa al control de la plusvalía por parte de los municipios, anunciada asimismo en el informe anual.

En términos de framing, existe un diagnóstico claro: la nueva ley afectaría a la clase media 
y, con ello, a una gran parte de la ciudadanía, con lo cual se posicionaba la medida como perjudicial para la sociedad. Habiendo salido como una propuesta del Presidente de la República, la atribución de responsabilidad estaba clara: el máximo mandatario estaba gobernando en contra de los intereses de una gran parte de la población.

Esta propuesta venía a fortalecer la idea que el diario privado venía manteniendo en sus informaciones y artículos de opinión durante todo el gobierno de Correa: el modelo económico del Gobierno no funciona. Además, servía para profundizar en la propuesta (tampoco nueva) de reducir el Estado, por cuanto "Alimentar la obesidad estatal con nuevos tributos no es una buena noticia" (Editorial El Comercio, 10/06/2015), con lo cual se estarían justificando las protestas ciudadanas. En suma, un gobierno alejado de la realidad estaría tomando medidas erróneas que perjudican a una gran parte de la población y que habrían generado una "legítima" (Editorial El Comercio, 25/06/2015) "oleada de manifestaciones ciudadanas en varias urbes del país" (Editorial El Comercio, 12/06/2015). Frente a este encuadre, los medios públicos proponían otro distinto:

Si ahora el Ejecutivo envía un proyecto de ley para frenar este enriquecimiento indebido e incremento del patrimonio por cuenta de unos cuantos 'sabidos', evidentemente habrá oposición y bastará con identificar quiénes salen al ruedo para entender qué fortunas están detrás para oponerse con falsos postulados (Editorial El Telégrafo, 28/05/2015).

Éstas serían las grandes fortunas enriquecidas durante años con información privilegiada y quienes proponen, desde la derecha, "el cero impuestos y el rentismo como paradigma social de la economía" (editorial El Telégrafo, 03/06/2015). Es, en definitiva, una medida ideológica que pretende crear una sociedad más justa y que desenmascara a los sectores que no están dispuestos a perder sus privilegios.

En términos de framing, la prensa pública propone que la medida supone un cambio a favor de los más pobres y, por eso, la reacción previsible de la clase privilegiada, que no está dispuesta a renunciar a su riqueza (construida en ocasiones de manera ilegítima" y va a "defender -al costo que sea- el statu quo" (Editorial El Telégrafo, 07/06/2015). De esta manera, nos encontramos frente a una lucha de encuadres contrapuestos, liderados por la prensa privada y los empresarios, por un lado, y la prensa pública y el gobierno, por el otro. Mientras los encuadres mediáticos estuvieron claros desde el principio, se sucedieron distintas reacciones desde los campos políticos y empresariales. El 30 de mayo de 2015, en el programa de rendición de cuentas semanal, Enlace Ciudadano, Rafael Correa realiza unas precisiones sobre la medida, indicando que se trata de un impuesto marginal y que afectaría a la vivienda, a fin de evitar la especulación. Estos ajustes continúan en el Enlace de la semana posterior:

Yo presenté un impuesto bastante progresivo con un impuesto mayor de $77,5 \%$. Salieron diciendo que era confiscatorio, como que si a todo se le iba a cobrar [esta tasa]. No nos coman al cuento. Por ejemplo, se paga 77,5\% desde $\$ 2$ adicionales a $\$ 849.600$. El último dólar paga $77,5 \%$. Todos los anteriores pagan una tasa inferior y los primeros pagan cero. El tipo promedio lo que 
efectivamente se paga impuesto no del tipo marginal, lo que está al margen y con eso nos engañaron [...] Esto no es para la clase media, es para las grandes fortunas (Rafael Correa, Enlace Ciudadano, 06/06/2015).

De esta manera, se diferenciaba entre un impuesto marginal y uno promedio, se presentaba una nueva tabla de cómo afectaría la medida según el valor de la vivienda y el expresidente la defendía aduciendo que quienes están en contra "están defendiendo las dinastías", en lo que proponía como una suerte de capitalismo anacrónico. El 13 de junio se anuncia que el impuesto máximo sería del $47,5 \%$ para herederos no directos y, finalmente, el expresidente paraliza el proyecto para dar inicio a un diálogo social: "Retiro de dos proyectos" (Editorial El Comercio, 16/06/2015) y anuncia el inicio de un "Diálogo Nacional" para profundizar en las medidas.

El siguiente cuadro sintetiza las funciones de los dos encuadres mediáticos en disputa durante ese período:

\begin{tabular}{|c|c|c|}
\hline Elemento del frame & El Comercio & El Telégrafo \\
\hline Diagnóstico & $\begin{array}{l}\text { Leyes Herencia y } \\
\text { Plusvalía afectan de } \\
\text { manera negativa a la } \\
\text { clase media }\end{array}$ & $\begin{array}{l}\text { Leyes Herencia y } \\
\text { Plusvalía ayudan a una } \\
\text { redestribución más justa } \\
\text { de la riqueza (beneficia a } \\
\text { los más pobres) y } \\
\text { suponen un cambio } \\
\text { social }\end{array}$ \\
\hline $\begin{array}{l}\text { Atribución de } \\
\text { responsabilidad }\end{array}$ & $\begin{array}{l}\text { Correa: modelo } \\
\text { (económico equivocado } \\
\text { "La ley de Herencias } \\
\text { tensó la cuerda y dividió } \\
\text { al país" (Editorial } \\
\text { El Comercio 26/05/2016) }\end{array}$ & $\begin{array}{l}\text { Derecha y sectores } \\
\text { privilegiados (burguesía), } \\
\text { que defienden su status } \\
\text { quo y sus privilegios a } \\
\text { cualquier precio }\end{array}$ \\
\hline Propuesta de solución & $\begin{array}{l}\text { Retirada definitiva de } \\
\text { proyecto }\end{array}$ & Aprobar la ley \\
\hline Atributos Correa & $\begin{array}{l}\text { Alejado de la realidad } \\
\text { Autoritario } \\
\text { Polarizante } \\
\text { Irresponsable } \\
\text { Deshonesto }\end{array}$ & $\begin{array}{l}\text { Responsable } \\
\text { Valiente }\end{array}$ \\
\hline $\begin{array}{l}\text { Atributos } \\
\text { manifestantes }\end{array}$ & $\begin{array}{l}\text { Sin violencia (salvo } \\
\text { excepciones) } \\
\text { "Protesta social legítima" } \\
(25 / 06 / 2016)\end{array}$ & $\begin{array}{l}\text { No son sólo movimiento } \\
\text { social, son líderes de la } \\
\text { oposición. } \\
\text { Violentos } \\
\text { Irresponsables } \\
\text { Electoralistas }\end{array}$ \\
\hline $\begin{array}{l}\text { Atributos } \\
\text { oposición }\end{array}$ & & $\begin{array}{l}\text { Irresponsables } \\
\text { Construyeron riqueza de } \\
\text { manera ilícita. Pretenden } \\
\text { privatizar lo público }\end{array}$ \\
\hline
\end{tabular}

Tabla 1. Elementos principales de los encuadres mediáticos durante las marchas de 2015. Fuente: Elaboración propia. 
Un año después, en el informe anual de mayo de 2016, el entonces Presidente Correa retoma la idea de la Ley de Herencias, no sin recibir la crítica de los medios privados: "La Ley de Herencias tensó la cuerda y dividió al país” (Editorial El Comercio, 26/05/2016). Finalmente, la ley es aprobada el 27 de diciembre de 2016 con algunos matices, lo que nos lleva a hablar de un proceso de "pseudopolítica" (Meyer, 2002), en el que durante un año se ha escenificado un ejercicio político en el que han participado otros actores (sociales y empresariales) para acabar aprobando lo que se propuso un año antes. La aprobación de la ley pasó sin apenas repercusión mediática, pues los medios, especialmente los privados, estaban ya más preocupados de la campaña electoral que iniciaba en enero de 2017.

\section{El paro nacional (2019)}

A pesar de que Lenín Moreno ganó las elecciones de 2017 de la mano de Rafael Correa, no tardó mucho en distanciarse del gobierno anterior, del que él mismo había formado parte, e iniciar un proceso de confrontación directa y de reconciliación con los grandes poderes. Tras la condonación de la deuda al sector financiero por un valor de 4.200 millones de dólares, el presidente Lenín Moreno, habiendo activado el discurso de la herencia recibida (articulándolo con una supuesta corrupción generalizada), firmó un crédito con el Fondo Monetario Internacional (FMI), que implicaba tomar medidas antipopulares. Fruto de este crédito, el 2 de octubre el presidente de la República emitió un decreto (Decreto 883) que significaba la toma de profundas medidas para la estructura económica del país.

En primer lugar, eliminaba los subsidios a los combustibles (gasolina y diésel); en segundo lugar, una reducción del Estado y afectación a los derechos laborales (23.000 despidos, $20 \%$ de reducción de salario para los nuevos contratos, disminución de las vacaciones de 30 a 15 días, flexibilización laboral o la obligatoriedad de trabajar un día gratis al mes, ajustes todos para el sector público). En tercer lugar, medidas que reducían la recaudación estatal (eliminación de aranceles) y, por último, acciones para proteger a las grandes fortunas (reducción del Impuesto a la Salida de Divisas o exenciones fiscales).

Este anuncio provocó, en primer lugar, las protestas de los transportistas, quienes rápidamente alcanzaron un acuerdo con el gobierno, pero las protestas se recrudecieron con la incorporación de otros sectores sociales: trabajadores, estudiantes y, sobre todo, indígenas. Después de 12 días de paro nacional y una fuerte represión policial que acabó con al menos 11 muertos, el gobierno se sentó en una mesa de negociación en la que se comprometió a sustituir el Decreto por otro.

Antes del anuncio de las medidas, la prensa privada ya venía advirtiendo de la necesidad de unos ajustes (sobre todo en la reducción del Estado) por la situación económica que habría generado el modelo económico del gobierno anterior de Rafael Correa. Sin embargo, no es hasta el 4 de octubre, cuando ya se había declarado el estado de excepción, cuando editorializa el tema: "el Gobierno no sólo tiene el derecho sino la obligación de tomar acciones para rescatar la economía de la ruinosa situación y acudir a medidas como la eliminación de subsidios [...]” (editorial El Comercio 04/10/2019), para luego 
fortalecerlo rápidamente: "Rechazo al vandalismo y oportuna acción militar" (editorial El Comercio 05/10/2019).

Inicialmente se identificaron como "marchas indígenas", aunque ya desde el principio se sugería una intervención de grupos correístas: "Es poderosamente curiosa, por no decir extraña, la convergencia de grupos perseguidos por el anterior Gobierno, con las consignas de los partidarios de la Revolución Ciudadana que rayan en desestabilización." (El Comercio 08/10/2019) y se hizo énfasis en el carácter vandálico de las protestas: "Los jueces deben actuar ante el vandalismo" (editorial El Comercio 09/10/2019) y se incidía en el "derecho a trabajar" de las personas frente al paro nacional y, a través de los artículos de opinión, se llegaba a cuestionar el derecho a la resistencia.

A pesar de que el paso de los días suponía un incremento en el número de manifestantes a lo largo de todo el país, el diario privado hablaba de una "minoría violenta" que paraliza la producción del país, al tiempo que lo caracterizaba como intento de golpe de Estado (11/10/2019). En el último día de paro, el diario privado hablaba de "hordas violentas bloquearon con palos y piedras los accesos y principales avenidas de Quito y los valles de los Chillos, Cumbayá y Tumbaco. A la par, asediaron urbanizaciones para amedrentar a los vecinos" (Editorial El Comercio, 13/10/2019). Mientras el foco se ponía en la supuesta violencia de los manifestantes, apenas o nada se decía de la declaración de toque de queda, de las muertes de manifestantes por heridas de bala de la policía, la detención de ciudadanos extranjeros o los ataques de la policía a los albergues establecidos como zona de paz en distintas universidades.

De esta manera, el encuadre del diario estaba claro: las medidas económicas son necesarias y quienes tratan de impedirlas son una minoría vandálica -en articulación con el corrupto gobierno anterior- que está perjudicando a todo el país provocando pérdidas milmillonarias. Todos los acontecimientos que no tuviesen cabida en este encuadre quedaban fuera de la cobertura mediática. De esta manera, se pasó de una minoría inicial a una conspiración preparada durante mucho tiempo que, según el diario, estaría intentando desestabilizar al gobierno y tras la que estaría el correísmo, que estaría utilizando una violencia terrorista (Editorial El Comercio, 14/10/2019 y 20/10/2019).

Este encuadre era reforzado también por las portadas del diario: "Estado de excepción ante el bloqueo y el vandalismo" (portada El Comercio 04/10/2019); "Vandalismo y bloqueo siguen; en vigencia toque de queda parcial" (portada El Comercio 09/10/2019) en las que se mostraban imágenes de los manifestantes cubiertos por el lanzamiento de gases lacrimógenos; "Radicalismo traba el diálogo y la economía se deteriora" (Portada El Comercio, 11/10/2019).

Por parte del diario público, El Telégrafo, encontramos una cobertura que respaldaba el discurso del propio gobierno, utilizando los mismos argumentos y datos: "se desmonta el subsidio a los combustibles" (Editorial El Telégrafo, 02/10/2019), "Eliminación de subsidios era una tarea pendiente" (Editorial El Telégrafo, 03/10/2019). Tras la identificación de los transportistas como un grupo poderoso y privilegiado que obtuvo prebendas en el gobierno de Correa (Editorial El Telégrafo, 04/10/2019) y la coincidencia con el diario privado en el derecho a llegar al trabajo de la ciudadanía, insiste en que la solución no pasa por paralizar el país (05/10/2019).

Si bien el diario público aborda el tema con una tibieza inicial, muestra una gran firme- 
za cuando las protestas se generalizan: "No al golpe de Estado", que atribuye a "quienes administraron antes el Estado" (editorial El Telégrafo 09/10/2019), en un cierre de filas total con el Gobierno:

Lo que ocurrió durante el sábado 12 de octubre responde a lo que en su momento advirtió el Presidente de la República: una confabulación tramada desde el extranjero, ejecutada por bandas terroristas y del narcotráfico, contrabandistas; pero también por quienes perdieron el poder y hoy son investigados por corrupción (Editorial El Telégrafo, 13/10/2019).

El recurso al discurso del presidente como argumentación del diario se convierte en una constante en la cobertura del paro, lo que da muestra del alto nivel de gubernamentalización del diario público en este período entendida la gubernamentalización como el uso de los medios públicos al servicio de los intereses del Gobierno.

La noche del 12 de octubre, como protesta al toque de queda decretado por el presidente, se produjo un cacerolazo masivo en el país, reacción que el diario público encuadró como un "cacerolazo en favor de la paz y en contra de los actos vandálicos" (editorial $E l$ Telégrafo 14/10/2019), en un intento de reescribir los events en favor del Gobierno. De la misma manera, a pesar de que se defiende la libertad de expresión en los editoriales, nada se menciona en ninguno de los dos diarios sobre el cierre de varios medios de comunicación que estaban informando sobre las protestas y la represión policial y militar. No en vano, y a pesar de que el diario público ofreció muy poca cobertura sobre los acontecimientos, durante los días del paro se produjo un cambio en la dirección del diario, que según algunas fuentes podría haberse debido a un intento de dar mayor cobertura a los hechos.

Las portadas del diario público mostraron el paro sólo de manera tangencial: "El estado de excepción arrancó a las 16:00 de ayer" (Portada El Telégrafo, 04/10/2019) y, cuando lo hace, refuerza el frame de los editoriales: "Quito y Guayaquil rechazan la violencia" (Portada El Telégrafo, 09/10/2019): "Moreno convoca a los sectores sociales ecuatorianos a un 'diálogo sincero"' (Portada El Telégrafo, 08/10/2019, con una imagen del presidente respaldado por la cúpula militar).

El siguiente cuadro resume los elementos de los encuadres de cada uno de los dos diarios durante las jornadas de paro nacional de 2019: 


\begin{tabular}{|c|c|c|}
\hline Elemento del frame & El Comercio & El Telégrafo \\
\hline Diagnóstico & $\begin{array}{l}\text { Las medidas tomadas } \\
\text { son adecuadas y } \\
\text { necesarias por la } \\
\text { situación económica que } \\
\text { ha provocado el gobierno } \\
\text { anterior }\end{array}$ & $\begin{array}{l}\text { Las medidas son } \\
\text { necesarias ante la crisis } \\
\text { provocada por el } \\
\text { gobierno anterior. } \\
\text { Medidas benefician a los } \\
\text { más pobres }\end{array}$ \\
\hline $\begin{array}{l}\text { Atribución de } \\
\text { responsabilidad }\end{array}$ & $\begin{array}{l}\text { Correa: situación } \\
\text { económica } \\
\text { Manifestantes: violencia }\end{array}$ & $\begin{array}{l}\text { Correa: situación } \\
\text { económica } \\
\text { Grupo privilegiado por } \\
\text { Correa (transportistas) } \\
\text { primero y, después, } \\
\text { violentos infiltrados del } \\
\text { gobierno anterior }\end{array}$ \\
\hline Propuesta de solución & $\begin{array}{l}\text { Mantener las medidas del } \\
\text { Decreto y ampliarlas }\end{array}$ & $\begin{array}{l}\text { Mantener las medidas y } \\
\text { dialogar }\end{array}$ \\
\hline Atributos de Moreno & $\begin{array}{l}\text { Rresponsable } \\
\text { Valiente }\end{array}$ & $\begin{array}{l}\text { Responsable } \\
\text { Valiente ("Mis opositores } \\
\text { están de acuerdo con } \\
\text { estas medidas en privado, } \\
\text { pero no se atreven a } \\
\text { decirlo en público", } \\
\text { editorial 3/10/2019) }\end{array}$ \\
\hline $\begin{array}{l}\text { Atributos de } \\
\text { manifestantes }\end{array}$ & $\begin{array}{l}\text { Vándalos } \\
\text { Infiltrados por miembros } \\
\text { del gobierno anterior }\end{array}$ & $\begin{array}{l}\text { Turbas } \\
\text { Infiltrados }\end{array}$ \\
\hline $\begin{array}{l}\text { Atributos de } \\
\text { oposición }\end{array}$ & $\begin{array}{l}\text { Correísmo ha orquestado } \\
\text { el intento del golpe de } \\
\text { Estado para desestabilizar } \\
\text { al gobierno. } \\
\text { Irresponsables. Violentos. } \\
\text { Corruptos. Terroristas }\end{array}$ & $\begin{array}{l}\text { Correísmo, en } \\
\text { conspiración con } \\
\text { Maduro, ha intentado } \\
\text { un golpe de Estado. } \\
\text { Corruptos.Violentos. } \\
\text { Terroristas. Cobardes. } \\
\text { Odiadores }\end{array}$ \\
\hline
\end{tabular}

Tabla 2. Elementos principales de los encuadres mediáticos durante el paro nacional de 2019. Fuente: elaboración propia.

Una vez se instala la mesa de negociación y se anuncia la derogación del decreto, el medio privado lo enfoca como un triunfo del gobierno, que tendió la mano al diálogo, indicando además que fue posible porque la dirigencia indígena se desmarcó del correísmo (editorial El Comercio, 14/10/2019). Por su parte, el diario público se centraba más en los destrozos patrimoniales causados por "actos vandálicos" y la vuelta a la calma y a la "paz" tras la destrucción y el odio generado por un grupo de "terroristas" que intentaron "desestabilizar al Gobierno" (editorial El Telégrafo 16/10/2019) en unas “jornadas de terror [...] que quedó registrada como una de las grandes tragedias vividas por Ecuador" (Editorial El Telégrafo, 21/10/2019).

Durante los días de paro nacional de 2019 se repitió un fenómeno que viene siendo habitual en el consumo de información: ante la falta de información ofrecida por los medios 
convencionales (que ocultaron los ataques de la policía a las zonas identificadas como zona de paz, a hospitales, la excesiva represión de las autoridades, la detención de ciudadanos de manera arbitraria, etc.), los ciudadanos optaron por informarse y convocarse a través de las redes sociales y los medios alternativos, que cumplieron un papel importante durante los días de paro nacional. Una vez se desconvoca el paro, el Gobierno anunció investigaciones contra estos medios por lo que consideró generación de noticias falsas.

\section{Conclusiones}

En este trabajo hemos partido de la propuesta de que, en ciertos momentos, se generan procesos de mediatización de la política que ilustran altos niveles de paralelismo político y que podrían estar influidos tanto por las características del sistema mediático como por la regulación del sector mediático, entre otros factores. A partir de ello, realizamos un análisis del comportamiento de dos medios de comunicación (público y privado) durante dos momentos de gran conflictividad social (2015 y 2019) a la luz de la teoría del framing.

Tal y como hemos podido comprobar y tal como se esperaba en nuestra primera hipótesis, observamos una lucha de encuadres durante 2015, en la que los medios privados actúan como oposición al gobierno de Correa en un proceso de mediatización en el que pretenden -e inicialmente logran- paralizar las leyes de Herencia y Plusvalía. En este proceso, el diario público reforzó el encuadre oficial, según el cual estas leyes sólo afectaban a una minoría privilegiada que intentaba defender su statu quo. En un primer momento, de esta lucha de encuadres salen victoriosos los medios privados, quienes, apoyando las marchas "legítimas", solicitan la paralización de las leyes. Un año más tarde, sin embargo, el gobierno de Correa aprueba las leyes sin apenas oposición ni cobertura mediática, pues los medios ya estaban trabajando el momento electoral que inicia en enero de 2017.

Si en 2015 los medios privados apoyaron las protestas, en 2019, a pesar de que las medidas suponían un fuerte ajuste para las clases populares, caracterizan las marchas y el paro nacional como actos vandálicos y terroristas, orquestados por el propio expresidente Correa en un intento de golpe de Estado. Este encuadre es compartido por el diario público, quien realiza un cierre de filas absoluto en un período que ilustró el nivel más alto de gubernamentalización desde la creación del diario público $(\mathrm{H} 2)$.

Vemos, por tanto, cómo el encuadre propuesto por el gobierno en 2019 se fortalece con la editorialización de los dos diarios estudiados, en una muestra de realineamiento de los medios de comunicación con el gobierno después de una década de disputa político mediática y tras una reforma a la política pública que podría estar explicando, al menos parcialmente, este retorno a la colaboración entre los grandes poderes. Empero, este retorno a la colaboración no impide que se siga manteniendo la conflictividad con el gobierno anterior, a quien se continúa responsabilizando de lo acontecido incluso varios años después de su salida. Este cambio de comportamiento de los (mismos) medios de comunicación en distintos momentos y ante diferentes Gobiernos -que no se puede des- 
vincular de las modificaciones en la normativa en lo referido a la comunicación mediática- es una muestra de la velocidad y la magnitud con la que se suceden los cambios en el contexto mediático no sólo en Ecuador, sino en América Latina, y, en consecuencia, un indicador de la necesidad de pensar en nuevos modelos complementarios a la propuesta clásica de Hallin y Mancini.

\section{Notas}

1. https://www.registroficial.gob.ec/index.php/registro-oficial-web/publicaciones/suplementos/item/12369-suplemento-al-registro-oficial-no-111

\section{Bibliografía}

Albuquerque, A. (2012). "O Paralelismo político em questão". Revista COM Política 2 (1). Chavero, P. (2015). Prensa y política en tiempos de crisis: estudio de la legislatura 2008-2011. Madrid: CIS.

Chavero, P. (2015b). "Intervención del Estado en Comunicación: políticas públicas para la democratización de la comunicación. Una aproximación al caso de Ecuador". ALCANCE, Revista cubana de información y comunicación 4 (8), pp. 3-25.

Chavero, P. y Oller, M. (2015). "Políticas públicas en comunicación y sistemas mediáticos. El caso de Ecuador". La Comunicación y el Periodismo de Ecuador frente a los desafíos contemporáneos. Cuadernos Artesanos de Comunicación $N^{\circ}$ 74. La Laguna.

Chavero, P. (2018). "Mediatización de la política en los nuevos gobiernos latinoamericanos: de la confrontación a la reconciliación”. Revista Internacional de Comunicación y Desarrollo No 8, pp. 119-128.

Entman, R. (1993). "Framing: toward clarification of a fractured paradigm". Journal of Communication 43 (4), 51-58.

Entman, R. (2004). Projections of power. Framing news, public opinion and US foreign policy. Chicago \& London: The University Chicago Press.

Fiss, O. (1997). Libertad de expresión y estructura social. México: Distribuciones Fontamara. Guerrero, M y Márquez, M. (2014). "El modelo "liberal capturado" de sistemas mediáticos, periodismo y comunicación en América Latina”. Temas de Comunicación. No 29, pp. 135-170.

Hallin, D. y Mancini, P. (2008). Sistemas mediáticos comparados. Barcelona: Hacer Editorial. Hallin, D. y Papathanassopoulos, S. (2002). "Political clientelism and the media: southern Europe and Latin America in comparative perspective". Media, Culture and society 24 (2), pp: 175-195.

Mazzoleni, G. y Schulz, W. (1999). "Mediatization of politics: a challenge for democracy?”. Political Communication 16, pp: 247-261.

McCombs, M. (2004). Estableciendo la agenda. Barcelona: Paidós Comunicación. 
Meyer, T. (2002). Media democracy: how the media colonize politics. Cambridge: Polity Press. Strömback, J. (2008). "Four Phases of Mediatization: An Analysis of the Mediatization of Politics”. Press/Politics 13 (3), pp: 228-246.

\begin{abstract}
The last decade in Ecuador was marked by the media-political dispute between the former President of the Republic, Rafael Correa, and the private media that became political actors. With the arrival in 2017 of the new president Lenín Moreno (a successor of Correa himself), Ecuador faced a change not only of leader, but also a sharp turn in national politics. The conservative political and economic forces, adversaries during Correa's presidency, became Moreno allies. In this context, the mass media also turned from being adversaries and contesting Correa's political field to become Moreno's allies.

This article presents a comparative analysis of the way in which two of the main media framed the social reactions to different economic measures: the inheritance and capital gains laws of Correa (2015) and the Decree 883 of Moreno (2019). It involves the analyze of two newspapers, El Telégrafo (public) and El Comercio (private), since they allow to identify the differences between the media based on their legal nature in each period.
\end{abstract}

Keywords: frames - political mediatization - mass media - protests - Ecuador.

Resumo: A última década no Equador foi marcada pela disputa política-mídia entre o então presidente da República, Rafael Correa, e a mídia privada, que se tornou ator político. Com a chegada em 2017 do novo presidente, Lenín Moreno (sucessor do próprio Correa), o Equador enfrentou uma mudança não apenas de líder, mas também de uma mudança acentuada na política nacional. As forças políticas e econômicas conservadoras, adversárias durante a presidência de Correa, tornaram-se aliadas de Moreno. Naquele turno, a mídia também passou de adversária e contestou o terreno político para Correa para se tornar aliada de Moreno.

Este artigo faz uma análise comparativa da maneira pela qual duas das principais mídias enquadraram (Entman, 1993) as reações sociais a diferentes medidas econômicas: as leis do Legado e do Ganho de Capital (2015) e o Decreto 883 de Moreno (2019). Analisar El Telégrafo (público) e El Comercio (privado) também nos permitirá identificar as diferenças entre os meios de comunicação com base em sua natureza jurídica em cada um dos períodos.

Palavras chave: frames - mediação da política - meios de comunicação de massa - protestos - Equador.

[Las traducciones de los abstracts fueron supervisadas por el autor de cada artículo] 